\title{
Helicobacter Pylori Outer Membrane Vesicles Bind To And Internalize Within Gastric Epithelial Cells
}

\author{
Heather Parker,* Dr Mark Hampton, ** Dr Jacqui Keenan* \\ *Departments of Surgery \& **Pathology, Christchurch School of Medicine and Health Sciences, PO \\ Box 4345, Christchurch, New Zealand
}

Helicobacter pylori are Gram negative spiral bacteria that inhabit the human stomach and are estimated to infect over half the world population [1]. Infection is always associated with gastritis, inflammation of the gastric tissue, and is also associated with the development of peptic ulcer disease or gastric cancer [2-3]. Indeed, H. pylori is the only bacterium to be awarded the status of Class 1 carcinogen by the World Health Organization [4].

H. pylori colonize this inhospitable niche by burrowing into the thick mucus overlying the gastric epithelium, where they are protected from the harsh acidic environment of the stomach. H. pylori are not invasive and reside over the surface of the gastric epithelium, preferentially above intracellular junctions [5]. Like many Gram negative bacteria, H. pylori bleb off part of their outer membrane as vesicles during growth. Little is known about these outer membrane vesicles (OMV), however, it has been suggested that they may act as a vehicle to transport virulence factors to the gastric mucosa [6]. It is our hypothesis that OMV interact directly with gastric epithelial cells, altering host intracellular signaling and production of inflammatory mediators. Recently, our group has demonstrated that OMV stimulate AGS cell (a gastric epithelial adenocarcinoma cell line) secretion of a proinflammatory chemokine interleukin-8 (IL-8) [7].

The small size (50-300 nm) of OMV does not permit their visualization by light microscopy [8]. Therefore, in order to follow their interaction with gastric epithelial cells, fluorescent and transmission electron microscopy techniques and flow cytometry were employed. The membrane dye, DiO, was used as a novel approach to labeling OMV. DiO-labeled OMV were observed in direct association with AGS cells by fluorescent microscopy (Figure 1 A), and flow cytometry showed vesicle to cell association increased over time. Trypan blue was used to quench extracellular $\mathrm{DiO}$ vesicle fluorescence to determine if OMV adhered to cells or were internalized. The addition of trypan blue did not decrease overall fluorescence, indicating that the majority of the vesicles were within the AGS cells (Figure 1 B). This was confirmed by immunogold labeling (Figure 2).

These results demonstrate that $H$. pylori OMV are internalized by AGS cells. Whether stimulation of IL-8 secretion by these cells is associated with OMV binding and/or internalization remains to be determined.

\section{References}

[1] J. Parsonnet, Aliment. Pharmacol. Ther. 9 (suppl. 2) (1995) 45.

[2] A. Lee, Scand. J. Gastroenterol. (suppl. 201) (1994) 2.

[3] J. Parsonnet, Environ. Health. Perspect. 103 (suppl. 8) 263.

[4] International Agency for Research on Cancer, Monograph 61 (1994). 
[5] S. L. Hazell and A. Lee, J. Infect. Dis. 153 (1986) 658.

[6] J. Keenan et al., FEMS. Microbiol. Lett. 182 (2000) 259.

[7] S. Ismail et al., Infect. Immun. 71 (2003) 5670.

[8] R. Fiocca et al., J. Pathol. 188 (1999) 220.

[9] This research was supported by a Top Achiever Doctoral Scholarship and the University of Otago, New Zealand.
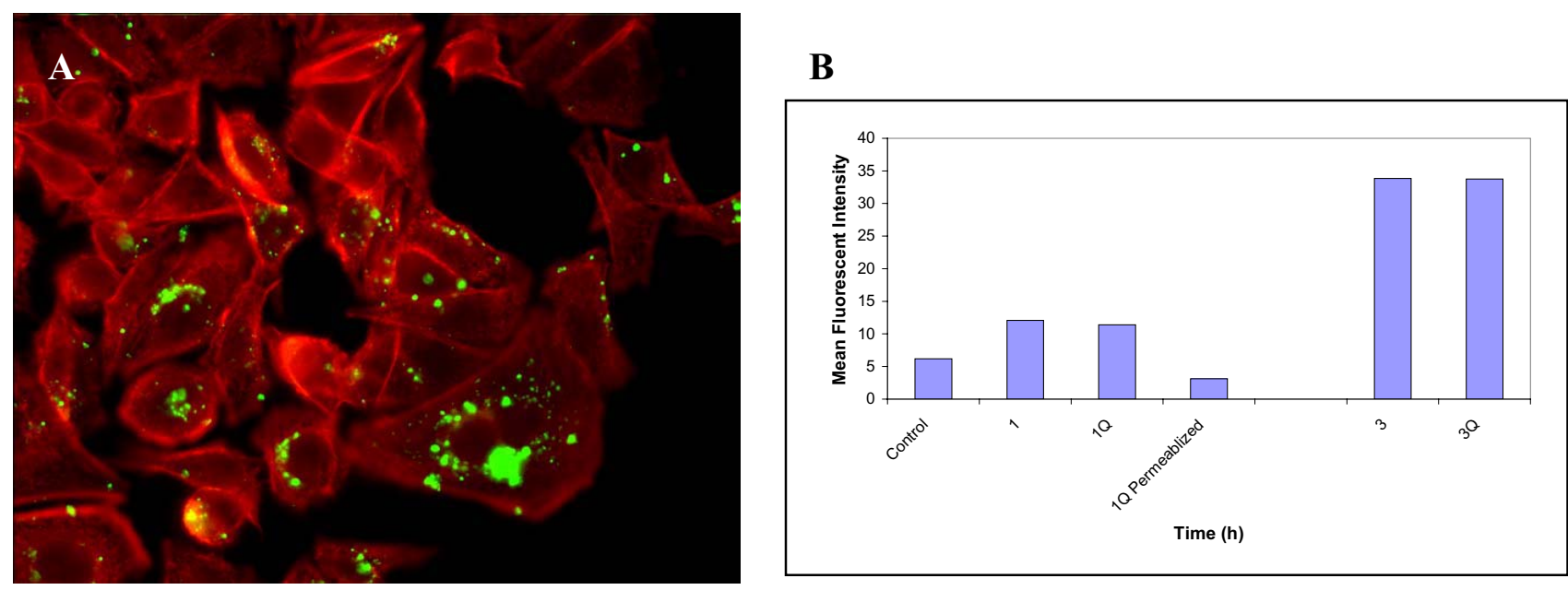

Fig. 1. H. pylori OMV are internalized by gastric epithelial cells. (A) Fluorescent microscopy shows OMV (green) associate with AGS cells (red). (B) This graph shows the mean fluorescent intensity (MFI) obtained in a representative experiment where AGS cells were incubated with DiO OMV for up to 3 hours. Some of the samples were treated with trypan blue (1Q \& 3Q). These show similar MFI to those not treated with trypan blue indicating that most of the vesicles are within the cells. Other samples were fixed and permeabilized then treated with trypan blue to confirm that this dye quenches $\mathrm{DiO}$ vesicle fluorescence. Control was AGS alone.
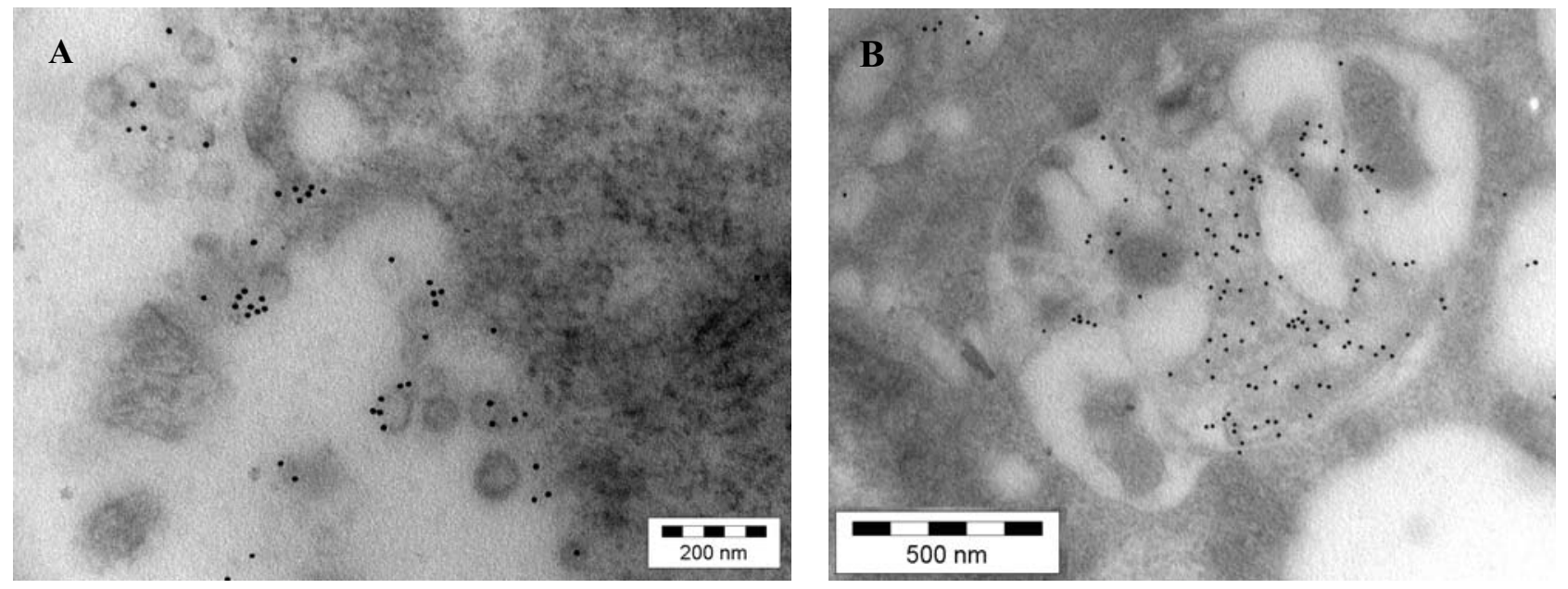

Fig. 2. After $24 \mathrm{~h}$ co-incubation of AGS cells with OMV, immunogold labeling shows aggregates of OMV (A) binding to gastric epithelial cell surfaces and (B) within cytoplasmic membrane bound organelles. 